\title{
Psoríase na infância
}

\author{
Psoriasis in childhood
}

Psoriasis en la infancia

Amanda Hertz*

\section{Resumo}

Psoríase é uma doença crônica, imuneinflamatória com prevalência de 1 a $2 \%$ na população pediátrica. ${ }^{1}$ A presença de lesões na pele pode significar um fator de baixa da autoestima e/ou dificuldade de convivência nas atividades escolares e sociais, causando impacto na qualidade de vida que pode ser significativo. Neste trabalho, será realizada uma revisão sobre psoríase na infância, considerando os aspectos epidemiológicos, clínicos, comorbidades associadas e terapêutica.

Descritores: Psoríase; Pediatria; Qualidade de vida.

\begin{abstract}
Psoriasis is a chronic, immune-inflammatory with a prevalence of 1 to $2 \%$ in pediatric population. ${ }^{1}$ The presence of skin lesions can mean a factor of difficulty in school co-existence and social activities, impacting on quality of life that can be significant. In this review on childhood psoriasis, epidemiological, clinical, and therapeutic aspects associated comorbidities are revised.
\end{abstract}

Keywords: Psoriasis; Pediatrics; Quality of life.

\section{Resumen}

La psoriasis es una enfermedad crónica, inmune-inflamatoria con prevalencia de 1 a $2 \%$ en la población pediátrica. ${ }^{1}$ La presencia de lesiones en la piel puede ser un factor de baja autoestima y dificultad para convivir en actividades escolares y sociales, impactando tal vez de forma significativa en la calidad de vida. En este trabajo se realizará una revisión sobre la psoriasis en la infancia. Considerando los aspectos: epidemiológicos, clínicos, comorbidades asociadas y terapeútica.

Palabras clave: Psoriasis; Pediatría; Calidad de vida. 


\section{Introdução}

Psoríase é uma doença crônica, imuneinflamatória, que acomete de 1 a $2 \%$ da população pediátrica. ${ }^{1}$

Poucos estudos epidemiológicos têm sido realizados em crianças e adolescentes. Até o momento, não há nenhuma revisão epidemiológica brasileira sobre este assunto. De acordo com a literatura, a psoríase na infância está associada a um maior risco de hiperlipidemia, obesidade, artrite reumatoide, hipertensão, diabetes mellitus e doença de Chron. ${ }^{2}$

O objetivo deste trabalho é realizar uma revisão sobre o perfil clínico, epidemiológico e associações de comorbidades da psoríase na infância e adolescência.

\section{Histórico}

A palavra psoríase provém do grego psora, que significa prurido. No entanto, até o século XVIII psoríase, hanseníase e outras entidades eram frequentemente classificadas em conjunto, e os doentes eram tratados com o mesmo preconceito e marginalização por parte da sociedade. Somente em 1841, a psoríase foi definida como uma entidade distinta, separada da hanseníase por Ferdinand von Hebra. ${ }^{3}$

\section{Epidemiologia}

A psoríase acomete de 1 a $2 \%$ da população pediátrica. ${ }^{1}$ Estima-se que de 25 a $45 \%$ dos casos de psoríase possam iniciar-se antes dos 16 anos e, em cerca de $2 \%$ dos casos, antes dos dois anos de vida. ${ }^{4}$

Embora tenha sido observada maior prevalência de psoríase em crianças do sexo feminino em alguns estudos, ${ }^{5}$ outros indicam que ambos os gêneros são afetados igualmente, como ocorre nos adultos. ${ }^{6,7}$

O risco de desenvolver psoríase é maior quando há história familiar. Se um dos pais é afetado, a chance de a criança desenvolver o quadro é de $14 \%$, sendo maior (41\%) quando acomete ambos. ${ }^{8}$ Estudos com pares de gêmeos demonstram uma concordância entre gêmeos monozigóticos de até $70 \%$, comparando entre 12 e $23 \%$ em gêmeos dizigóticos. ${ }^{7}$

\section{Etiopatogenia}

Apesar dos avanços na última década, a causa da psoríase permanece desconhecida. Trata-se de doença da pele e das articulações, com predisposição poligênica. No passado, considerava-se o distúrbio dos queratinócitos como a base etiopatogênica da psoríase; entretanto, atualmente, sabe-se que é uma alteração inicialmente imunológica, mediada pela resposta tipo Th1. ${ }^{6,9,10}$

O componente genético envolvido na etiologia da psoríase pode ser evidenciado por estudos sobre a incidência familiar, concordância entre gêmeos e identidade de antígenos de histocompatibilidade (HLA). Múltiplos alelos HLAs têm sido associados à psoríase, especialmente: HLACw6, HLA-B13, HLA-B17, HLA-B37, HLA-DR7, HLA-B46, HLA-B57, HLA-Cw1 e HLA-DQ9. Estudos recentes revelam loci de susceptibilidade no cromossomo 6p, 17q, 4q, 1q e 3q designados, respectivamente, como Psors1, Psors2, Psors3, Psors4 e Psors5 .6,7

A imunopatogênese da psoríase é complexa e envolve alterações do sistema imunológico inato (queratinócitos, células dendríticas - CD -, macrófagos, neutrófilos, mastócitos, células endoteliais) e adquirido (linfócitos T). ${ }^{1}$ As células do sistema imune inato (SII) ativadas produzem fatores de crescimento, citocinas e quimiocinas, que atuam sobre as células do sistema imune adquirido (SIA) e vice-versa. ${ }^{1-5}$ Inicialmente, ocorre ativação das células do SII (CD e queratinócitos). Vários fatores ambientais, como o trauma mecânico, infecções, medicamentos e estresse emocional são considerados desencadeantes da doença. Uma vez ativada, a CD processa um antígeno (ambiental ou endógeno, ainda não definido) e migra para o linfonodo regional, onde o apresenta aos linfócitos T (LT). Os LT ativados dos doentes com psoríase diferenciam-se, preferencialmente em LTCD4 tipo1 (LTh1; produtores de INF- $\gamma$, TNF- $\alpha$ e IL-2) e tipo 17 (LTh17; produtores de IL-17, TNF- $\alpha$, IL-6; IL-22) e LTCD8 ${ }^{+}$tipo 1 (LTc1; produtores de TNF- $\alpha$, INF- $\gamma$, perforinas e granzima B). ${ }^{11-15}$

\section{Aspectos clínicos}

A anamnese deve ser completa. É importante ressaltar os fatores precipitantes e exacerbadores da psoríase: infecções estreptocócicas, medicações (antimaláricos, lítio, betabloqueador), 
trauma, estresse emocional. ${ }^{16,17}$

Quanto à forma clínica, a psoríase em placas é a variante clínica mais comum em crianças e adolescentes (de 34 a 84\%). ${ }^{6}$

As lesões configuram-se por pápulas e placas eritematosas com descamação esbranquiçada, bem delimitadas, de tamanhos variados, frequentemente de maneira simétrica. Na infância, a psoríase pode apresentar características atípicas, como placas com padrão folicular ou acometendo áreas como a região da face - incluindo a porção periorbitária, perioral e nasal - e dificultando o diagnóstico, muitas vezes ${ }^{18}$ (Figura 1).

As lesões de psoríase afetam, em geral, o couro cabeludo (Figura 3), seguido da superfície extensora das extremidades e tronco. Ocorre, frequentemente, distribuição simétrica das lesões e ausência de prurido. $\mathrm{O}$ acometimento das mãos, dos pés, da genitália e das áreas flexurais, inclusive periumbilical, também é mais comum em crianças. ${ }^{7,19,20}$

O acometimento do couro cabeludo, com a presença de escamas brancas, aderentes e espessas ao redor dos folículos pilosos com leve eritema (pseudotinea amiantacea), pode levar à queda dos cabelos. Pode ocorrer lesão única em placa ou mesmo lesões mal delimitadas e descamativas, clinicamente indistinguíveis da dermatite seborreica. ${ }^{19,20}$
A psoríase gutata é a segunda variante clínica mais comum, aparece de forma abrupta e é precedida, geralmente, por uma infecção estreptocócica (de 56 a 85\% dos casos). ${ }^{6}$ Lesões ovais de 2 a 6 $\mathrm{mm}$ de diâmetro estão dispostas simetricamente em toda a superfície corpórea, predominando no tronco e na raiz dos membros. Embora a psoríase gutata possa desaparecer espontaneamente, 40\% das crianças evoluem para o tipo placa., ${ }^{1721}$

Psoríase na área de fraldas, acomete crianças de até dois anos. As lesões apresentam placas eritematosas e brilhante, bordas bem delimitadas e envolvimento das dobras inguinais (diferente da dermatite de contato por irritante primário), com prurido variável. ${ }^{22}$ Classicamente, tais sinais e sintomas respondem muito pouco ao tratamento convencional para dermatite de fraldas. ${ }^{20} \mathrm{~A}$ psoríase nessa área está correlacionada com efeito Koebner, devido ao trauma por exposição de fezes e urina, e geralmente há melhora após a retirada de fraldas.

A psoríase pustulosa é rara e considerada a forma mais grave, ocorrendo em aproximadamente $1 \%$ da população pediátrica portadora de psoríase. Caracteriza-se por múltiplas pústulas, estéreis, sobre base eritematosa, podendo ser generalizada ou localizada. A forma generalizada (von Zumbusch) pode ser desencadeada em um paciente com psoríase vulgar por interrupção

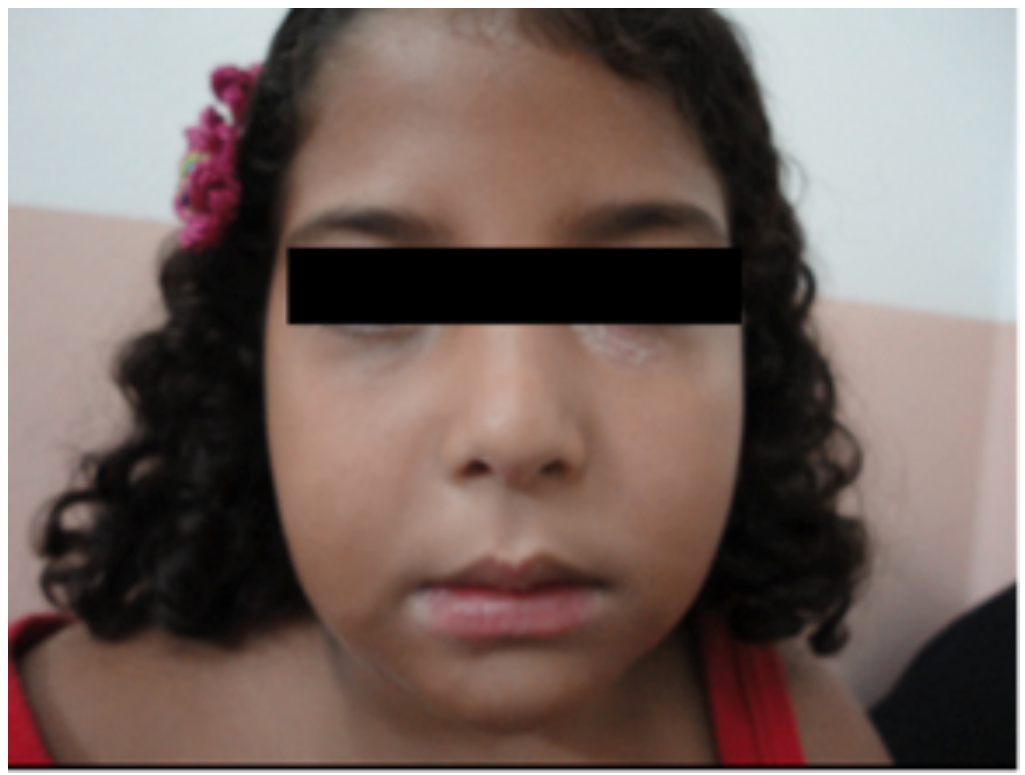

Figura 1. Acometimento periorbital.

Fonte: Instituto de Dermatologia Prof. R. D. Azulay - Dra. Amanda Hertz. 


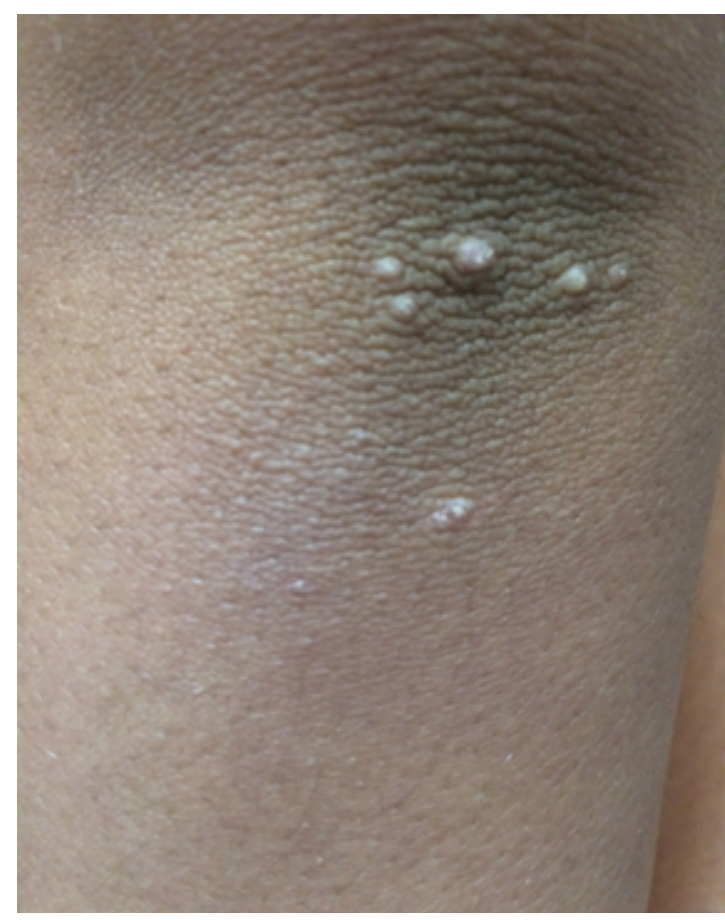

Figura 2. Psoríase de padrão folicular. Fonte: Hospital Universitário Pedro Ernesto - Dra. Luna Azulay. do corticoide sistêmico, por hipocalcemia, por infecção ou por irritantes locais. Frequentemente, há comprometimento do estado geral, febre alta e leucocitose. A forma localizada compreende três subformas: psoríase pustulosa em placas ou anular, acrodermatite contínua de Hallopeau (pústulas ou lagos de pus nos quirodáctilos e, mais raramente, dos pododáctilos, de curso crônico, sem tendência a remissão espontânea) e pustulose palmoplantar (caracterizada por surtos de pústulas estéreis que comprometem palmas e/ ou plantas, simetricamente, sem manifestações). ${ }^{23}$ Complicações raras dessa forma de psoríase, descritas em crianças, são insuficiência renal, icterícia colestática, lesões líticas ósseas e osteomielite multifocal estéril. A forma anular, que associa lesões eritematodescamativas e pústulas periféricas, parece ser exclusiva da infância. Essa variante pode ser acompanhada de febre, osteomielite asséptica e comprometimento pulmonar. ${ }^{24}$ Psoríase pustulosa também pode estar associada com síndrome Sapho (sinovites, acne, pustulose palmoplantar e psoríase, hiperostose e osteíte). ${ }^{25}$

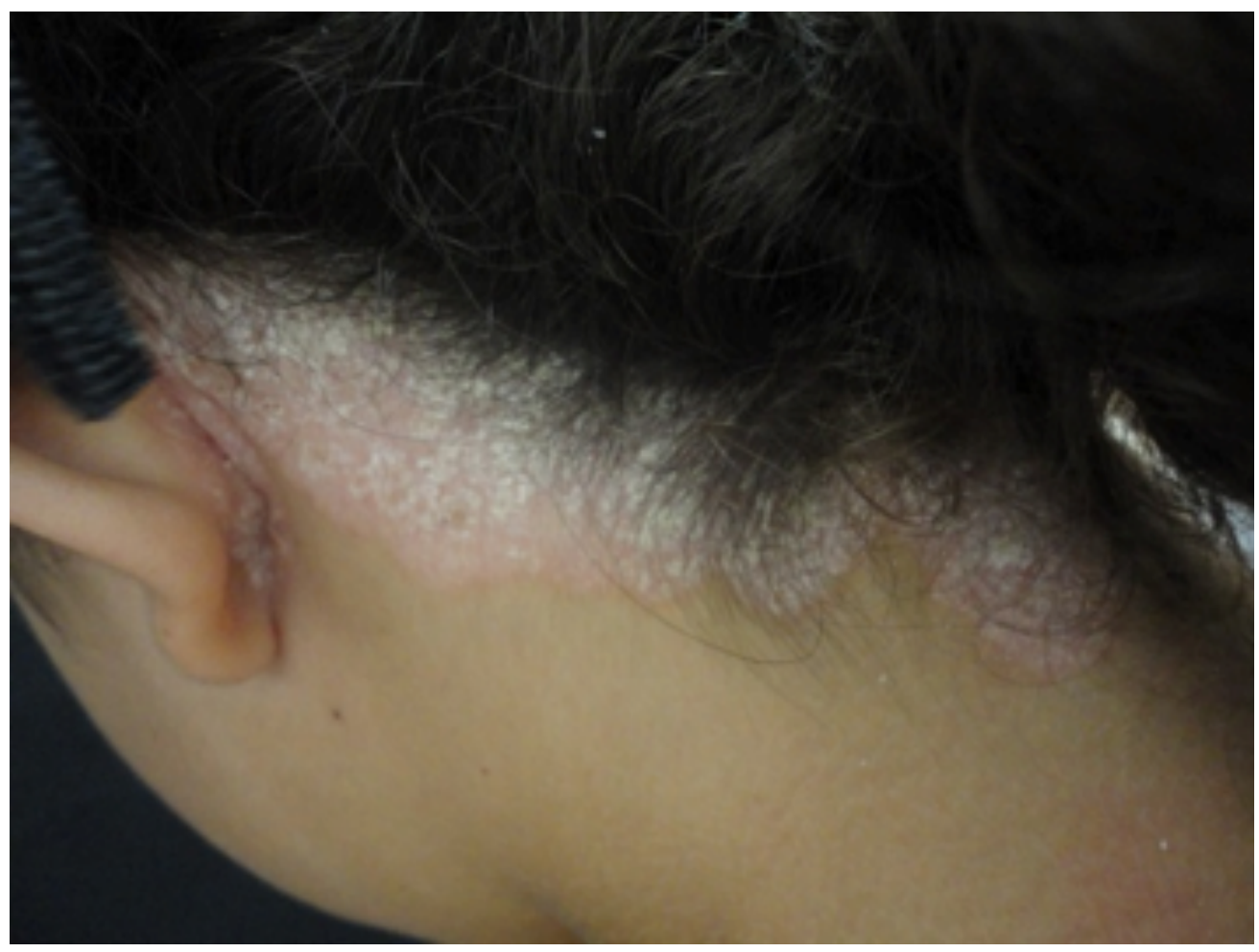

Figura 3. Acometimento no couro cabeludo.

Fonte: Instituto de Dermatologia Prof. R. D. Azulay - Dra. Amanda Hertz. 
Outras formas raras de psoríase são: eritrodérmica, linear e congênita. Na psoríase eritrodérmica há acometimento de mais de $90 \%$ da superfície corpórea, pouca descamação, além de ser extremamente rara. Pode ocorrer hipertermia ou hipotermia e, em casos de longa duração, pode haver diminuição do débito cardíaco e comprometimento das funções hepática e renal. O iminente risco de choque cardiovascular e mesmo séptico torna esses doentes extremamente graves, demandando imediata internação e pronta-intervenção terapêutica associada a medidas de suporte. ${ }^{24,26} \mathrm{~A}$ forma congênita é definida como a ocorrência de quaisquer variantes clínicas da psoríase ao nascimento ou durante os primeiros dias de vida. De acordo com a literatura, expressa-se na forma de psoríase em placas com envolvimento de face, couro cabeludo, tronco e poupando nádegas. ${ }^{27}$ Casos de psoríase eritrodérmica congênita ou neonatal são ainda mais raros, com apenas 15 casos na literatura. ${ }^{24}$

A psoríase linear é assunto controverso, caracteriza-se por lesões eritematoescamosas, seguindo as linhas de Blaschko. Pode ter início na infância ou na idade adulta e acometer, essencialmente, tronco ou membros, com extensão e progressão variáveis. Deve ser diferenciada do nevo epidérmico verrucoso inflamatório linear (Nevil), o qual é mais resistente à terapêutica. ${ }^{7}$

A artrite psoriásica caracteriza-se por uma forma de artrite soronegativa, encontrada entre 5 e $42 \%$ dos doentes de psoríase, sendo rara na infância. O pico de incidência é bifásico, geralmente um pico entre os dois anos de vida e o outro está entre 9 e 12 anos. $O$ sexo feminino é ligeiramente mais acometido. ${ }^{28}$

A forma mais frequente de artrite psoriásica é a monoartrite ou oligoartrite assimétrica, que afeta, principalmente, articulações das mãos e dos pés. Outras formas menos comuns apresentam comprometimentos simétrico, axial e, até mesmo, mutilante. Os quadros cutâneo e articular não têm relação do ponto de vista de atividade e evolução. A uveíte psoriásica, uma forma de uveíte anterior assimétrica, ocorre entre 14 e $17 \%$ de crianças com artrite psoriásica juvenil. ${ }^{28,29}$

Alterações ungueais são observadas entre 17 e $29 \%$ das crianças com psoríase. ${ }^{30}$ Pittings ungueais, por acometimento da matriz próxima, são as alterações mais frequentes. Onicólise, hi- perqueratose subungueal e "manchas de óleo" são outras alterações evidenciadas e correspondem ao acometimento do leito ungueal. O comprometimento ungueal pode aparecer isoladamente, preceder, coincidir ou aparecer após as lesões cutâneas de psoríase. ${ }^{31}$

\section{Diagnóstico}

O diagnóstico da psoríase é eminentemente clínico. Em caso de dúvida diagnóstica, pode ser realizado estudo histopatológico. Diagnósticos diferenciais a serem considerados na infância e na adolescência incluem: dermatite seborreica, eczemas, micoses superficiais, sífilis secundária, pitiríase rubra pilar, líquen plano, lúpus eritematoso, pitiríase liquenoide crônica, nevil, acrodermatite enteropática, pênfigo foliáceo eritrodérmico, eritrodermia por drogas, pustulose subcórnea de Sneddon-Wilkinson, pustulose exantemática aguda generalizada e impetigo bolhoso ${ }^{23}$ (Quadro 1).

\section{Comorbidades}

A psoríase na infância está associada a um maior risco de hiperlipidemia, obesidade, artrite reumatoide, hipertensão, diabetes mellitus, síndrome metabólica e doença de Chron. ${ }^{2}$

A presença de lesões na pele pode significar um fator de baixa da autoestima, dificuldade de convivência nas atividades escolares e sociais, causando impacto na qualidade de vida, que pode ser significativo para o seu desenvolvimento. Há relato que a qualidade de vida de crianças com psoríase é pior do que a de portadoras de diabetes e epilepsia. ${ }^{33}$

\section{Tratamento}

Na maioria dos doentes pediátricos, a psoríase pode ser tratada com medicações tópicas. A fototerapia é opção para casos mais extensos e refratários. A terapia sistêmica é reservada para casos graves e extensos, sem controle com tratamento tópico e/ou fototerapia. Sendo assim, as terapêuticas específicas dependerão da forma e da extensão da doença. ${ }^{34}$

\section{Tratamento tópico}

Emolientes deverão ser incluídos em qualquer 
Quadro 1. Formas clínicas e principais diagnósticos diferenciais da psoríase na infância e na adolescência. Fonte: Romiti et al., 2009.32

\begin{tabular}{|c|c|}
\hline Forma clínica & Diagnósticos diferenciais \\
\hline Psoríase em placas & $\begin{array}{l}\text { Eczema numular } \\
\text { Dermatite Seborreica } \\
\text { Tinea corporis }\end{array}$ \\
\hline Psoríase no couro cabeludo & $\begin{array}{l}\text { Tinea capitis } \\
\text { Dermatite atópica } \\
\text { Dermatite Seborreica }\end{array}$ \\
\hline Psoríase na área de fralda & $\begin{array}{l}\text { Acrodermatite enteropática } \\
\text { Candidáse } \\
\text { Dermatite de contato }\end{array}$ \\
\hline Psoríase nas flexuras & $\begin{array}{l}\text { Candidíase } \\
\text { Eritrasma } \\
\text { Tinea corporis } \\
\text { Dermatite de Contato }\end{array}$ \\
\hline Psoríase gutata & $\begin{array}{l}\text { Líquen plano } \\
\text { Pitiríase rósea } \\
\text { Pitiríase rubra pilar } \\
\text { Pitiríase liquenóde crônica } \\
\text { Sífilis secundária }\end{array}$ \\
\hline Psoríase pustolosa (generalizada) & $\begin{array}{l}\text { Síndrome da pele escaldada estafilocócica } \\
\text { Pustulose exantemática generalizada aguda }\end{array}$ \\
\hline Psoríase pustulosa (localizada) & $\begin{array}{l}\text { Tinea corporis } \\
\text { Síndrome de Sweet } \\
\text { Eritema anular centrífugo }\end{array}$ \\
\hline Psoríase pustulosa (palmoplantar) & $\begin{array}{l}\text { Disidrose infectada } \\
\text { Tinea manum }\end{array}$ \\
\hline Psoríase ungueal & $\begin{array}{l}\text { Onicomicose } \\
\text { Onicodistrofia } \\
\text { Líquen plano }\end{array}$ \\
\hline
\end{tabular}

esquema terapêutico, seja como coadjuvantes, seja em alternância com os produtos ativos, ou, até mesmo, em fases assintomáticas. As opções de uso tópico são as seguintes.

- corticosteroides tópicos: é a terapêutica tópica mais utilizada nos casos de psoríase infantil com poucas lesões. Apresentam ação anti-inflamatória, antiproliferativa e antipruriginosa. A eficácia da resposta aos corticosteroides tópicos varia conforme a forma clínica, sendo alta na psoríase invertida, moderada na psoríase do corpo e discreta na psoríase palmoplantar e ungueal. A localização das lesões de psoríase determina a potência dos corticosteroides tópicos a serem utilizados; corticoides de alta potência são utilizados em áreas mais espessas como couro cabeludo, palmas e plantas, enquanto corticoides de baixa potência, em áreas mais finas. Efeitos colaterais incluem: telangectasias, atrofia, estrias. Uso intermitente minimiza o risco de efeito colateral $;{ }^{18}$

- calcipotriol: análogo da vitamina D3, que diminui a proliferação e induz diferenciação dos queratinócitos, além de modificar a resposta imune. Geralmente mais efetivos quando combinados com corticoterapia tópica, permite períodos de remissão mais prolongados. A eficácia e a segurança do calcipotriol no tratamento de pacientes pediátricos ainda não estão bem-estabelecidas. Em diferentes relatos da literatura, o calcipotriol pomada tem se mostrado eficaz, bem 
tolerado e seguro em crianças com psoríase, sendo a irritação local o efeito colateral mais comumente relatados. Embora não existam guidelines formais para uso em crianças, o uso de até $45 \mathrm{~g} / \mathrm{sem} / \mathrm{m}^{2}$ em crianças parece não influenciar os níveis séricos de cálcio. ${ }^{35}$ Irritação, particularmente na face e em áreas intertriginosas, ocorre em mais de $20 \%$ dos pacientes. De acordo com Jager e colaboradores, o calcipotriol é considerado a primeira escolha de tratamento tópico em crianças (exceto em face e dobras). Grau de recomendação A: 155 pacientes tratados com calcipotriol. Eficaz, bem tolerado para crianças com psoríase em placas; ${ }^{36}$

- imunomoduladores tópicos: pimecrolimus e tacrolimus podem ser indicados para formas localizadas na face e dobras, por provocarem menos efeitos colaterais do que os corticosteroides e análogos da vitamina D e apresentam melhor absorção nessas áreas. A sua eficácia é extremamente variável. No Brasil, o pimecrolimus é indicado em crianças a partir de três meses de vida, e o tacrolimus, a partir de dois anos. ${ }^{37}$ De acordo com a revisão de Jager, o tacrolimus apresenta grau de recomendação C: tacrolimus $0,1 \%$ parece ser efetivo e seguro para curto período de tratamento em área intertriginosa e face. Longo período de segurança não foi descrito. Nenhuma conclusão do uso de pimecrolimus para psoríase devido ao pequeno número de pacientes (dois relatos de caso); ${ }^{36}$

- coaltar (de 2 a 10\%): uma das modalidades terapêuticas mais antigas, apresenta ação antiproliferativa e antipruriginosa. Quando em uso isolado, tem ação moderada na psoríase em placas, mas, quando associado à fototerapia, sua ação é potencializada. Pode ser combinado com ácido salicílico de 2 a 5\% em lesões hiperqueratósicas. A foliculite é o efeito colateral mais frequente da utilização do coaltar. Entre os inconvenientes do seu uso, pode-se citar a baixa aceitação cosmética. Existe conflito acerca da potencial carcinogenicidade do coaltar. Apesar de estudos in vitro e estudos em animais demonstrarem claramente seu potencial carcinogênico, estudos epidemiológicos com o uso de coaltar em seres humanos não demonstram aumento da incidência de neoplasias no grupo estudado; ${ }^{38}$

- antralina (ou ditranol): Apresenta potencial anti-inflamatório e antiproliferativo. Pode ser usada em baixas concentrações (de 0,1 a $0,5 \%$ ) durante 24 horas ou em altas concentrações (de 1 a 3\%) em aplicações de apenas 15 a 30 minutos. Preparado em creme, pasta ou pomada. O clareamento costuma ocorrer em três a quatro semanas. Substância irritante deve ser evitada em áreas intertriginosas, em área próxima aos olhos e mucosas e em pele sã perilesional, onde pode determinar erosões e bolhas. A antralina mancha roupas, azulejos e a pele ao redor das lesões. Praticamente, não há risco de toxicidade sistêmica, apresentando excelente perfil de segurança na faixa pediátrica. ${ }^{37}$

\section{Fototerapia e tratamento sistêmico}

Fototerapia com Puva, UVB banda larga (290$320 \mathrm{~nm}$ ) e banda estreita (311 nm). Trata-se de opção terapêutica utilizada de modo isolado ou combinado a outras modalidades terapêuticas, tópicas ou sistêmicas. O mecanismo de ação da fototerapia se faz mediante as atividades antiproliferativa, anti-inflamatória e imunossupressora. $\mathrm{O}$ risco relativo de carcinoma espinocelular na pele se eleva em pacientes expostos a altas dosagens de UV, definido como pelo menos 200 sessões ou $2.000 \mathrm{~J} / \mathrm{cm}^{2}$ de Puva ou 300 sessões de UVB, e esse risco continua elevado mesmo na década após a descontinuação do tratamento. ${ }^{18}$ Puva não é recomendado para menores de 12 anos. ${ }^{36}$

- antibióticos: embora não haja evidências de que a antibioticoterapia altere a evolução natural da psoríase gutata desencadeada por infecção, crianças com tal forma da doença e infecção estreptocócica documentada podem receber penicilina ou eritromicina por sete a 14 dias; ${ }^{24}$

- acitretina: derivado da vitamina A (retinol), é empregado na dose entre 0,25 e 1,0 mg/ kg/d. Representa a opção terapêutica sistêmica mais utilizada em crianças com quadros extensos e refratários a tratamento tópico e/ ou fototerapia. Os efeitos colaterais incluem: 
queilite leve (dose-dependente), epistaxe, conjuntivite, paroníquia, alopecia, prurido, dislipidemia e teratogenia (o etretinato persiste no organismo por dois anos, devendo ser, portanto, contraindicado em mulheres em idade fértil). A terapia prolongada com acitretina deve ser avaliada com cuidado em crianças, pois há relatos de fechamento prematuro das epífises ósseas, calcificações de tendões e ligamentos e retardo do crescimento ósseo. Exames radiológicos devem ser realizados anualmente. O risco do fechamento prematuro das epífises ósseas, uma preocupação em crianças, pode ser reduzido, como uso da menor dose eficaz possível (em geral entre 0,25 e $0,6 \mathrm{mg} / \mathrm{kg} / \mathrm{dia}$ ), ${ }^{18}$ desde que possa ser empregado até $1 \mathrm{mg} / \mathrm{kg} /$ dia (Consenso de psoríase, 2009). Entre as contraindicações absolutas, estão gestação ou desejo de engravidar nos próximos anos, insuficiência hepática e renal e alergia ao parabeno contido nas cápsulas. Não é contraindicado o uso concomitante de vacinas próprias da faixa etária. ${ }^{39}$ Aconselha-se o uso de retinoides para psoríase pustular, eritrodérmica ou refratária em crianças e adolescentes homens por curto período; ${ }^{18}$

- metotrexato: antagonista do ácido fólico. Pode ser administrado por via oral, intramuscular e endovenosa. O metotrexato deve ser empregado em casos extensos e resistentes de psoríase na infância ou em casos de psoríase artropática. A dose utilizada para doentes pediátricos é de 0,2 a $0,4 \mathrm{mg} / \mathrm{kg} /$ semana, até a dose total semanal de 12,5 a $20 \mathrm{mg}$. Pode ser associado ao ácido fólico. Apresenta rápido início de ação. A criança mais jovem tratada na literatura tinha quatro anos de idade e psoríase grave desde os dois anos de idade. Controles hematológicos e provas periódicas das funções hepática e renal são indispensáveis. Clinicamente, um dos sinais mais precoces de intolerância é o aparecimento de lesões aftoides na mucosa oral, traduzindo leucopenia importante. O efeito colateral mais comum é a intolerância gástrica. Vacinas de vírus vivos ou atenuados devem ser evitadas; ${ }^{18,37}$
- ciclosporina A: atua inibindo os linfócitos TCD4 ativados, impedindo a liberação de IL2. Embora tenha sido muito estudada em doentes com dermatite atópica, não há estudos de segurança e eficácia suficientes para psoríase em crianças. Deve ser reservada para casos graves, como psoríase eritrodérmica, e para casos rapidamente progressivos e sem resposta a outros métodos terapêuticos. A dose da ciclosporina é de 2 a $5 \mathrm{mg} / \mathrm{kg}$, diariamente, por três a quatro meses, quando deve ser gradualmente retirada. Os efeitos colaterais incluem nefrotoxicidade, hipertensão, náusea, sensações parestésicas, hiperplasia gengival, hipertricose e aumento do risco de neoplasias, porém não parecem mais frequentes em crianças quando comparados a adultos com psoríase. Para evitar dano renal em crianças obesas, a dose deve ser preferencialmente baseada no peso ideal do que real. A medicação requer monitorização renal, hematológica e hepática. As contraindicações ao uso de ciclosporina são anormalidades na função renal, hipertensão arterial sistêmica não controlada, malignidades. A imunização com vírus vivos ou atenuados deve ser evitada durante o período de tratamento e entre três e 12 meses após o seu término, dependendo da dose empregada; ${ }^{28}$

- imunobiológicos: medicamentos que interferem de maneira específica e pontual no sistema imune. Atuam bloqueando ou estimulando uma ou mais vias da resposta imunológica. Até o momento, o etanercepte é o biológico mais criteriosamente avaliado para o uso em crianças com psoríase ${ }^{40}$ Em recente estudo da literatura, crianças entre quatro e 17 anos com psoríase moderada a grave responderam de forma favorável à medicação na dose de $0,8 \mathrm{mg} / \mathrm{kg} / \mathrm{semana}$ (máximo de $50 \mathrm{mg}$ ), administrada por via subcutânea, no total de 48 semanas, com ocorrência de quatro eventos adversos sérios (incluindo pneumonia, gastroenterite, desidratação e remoção cirúrgica de cisto ovariano) que se resolveram sem sequelas. ${ }^{40,41}$ Etanercept é aprovado na Europa para tratamento de psoríase em crianças maiores de oito anos, mas ainda não é aprovada pelo FDA nos Estados 
Unidos da América. Infliximab apresenta estudos em crianças com Cronh e Adalimumab em crianças com artrite idiopática juvenil. ${ }^{42,43}$

\section{Conclusão}

A psoríase é uma doença inflamatória crônica, que acomete pele e articulações, com implicações significativas nos aspectos físico e emocional, ocasionando impacto importante na qualidade de vida dos pacientes. Há relatos de que a qualidade de vida de crianças com psoríase é pior do que de portadoras de diabetes e epilepsia. ${ }^{44}$ Devido ao caráter crônico da psoríase e suas comorbidades, mais estudos são necessários para avaliar estes pacientes a longo prazo.

\section{Referências}

1. Goldminz AM, Buzney CD, Kim N, Au SC, Levine DE, Wang AC, et al. Prevalence of the Metabolic Syndrome in children with psoriatic disease. Pediatr Dermatol. 2013;30(6):700-5.

2. de Jager ME, van der Kerkhof PC, de Jong EM, Seyger MM. A cross-sectional study using the Children's Dermatology Life Quality Index (CDLQI) in childhood psoriasis: the negative effect on the quality of life and moderate correlation of CDLQI with severity scores. Br J Dermatol. 2010;163(5):1099-1101. doi: 10.1111/j.1365-2133.2010.09993.x.

3. Schön MP, Boehncke WH. Psoriasis. N Engl J Med. 2005;353(8):848-50.

4. Watson W, Cann HM, Farber EM, Nall ML. The genetics of psoriasis. Arch Dermatol. 1972;105:197-207.

5. Tollefson MM, Crowson CS, McEvoy MT, Kremers HM. Incidence of psoriasis in children: a populationbased study. J Am Acad Dermatol. 2010;62:979-87.

6. Carneiro SCS. Psoríase: mecanismos de doença e implicações terapêuticas [tese]. São Paulo: Faculdade de Medicina da Universidade de São Paulo; 2007.

7. Benoit S, Hamm H. Childhood psoriasis. Clin Dermatol. 2007;25:555-62.

8. Andressen C, Henseler T. Inheritance of psoriasis: analysis of 2035 family histories. Hautarzt. 1982;(33):214-7.

9. Ghoreschi K, Weigert C, Röcken M. Immunopathogenesis and role of $\mathrm{T}$ cells in psoriasis. Clin Dermatol. 2007;25:574-80.

10. Sabat R, Philipp S, Höfflich C, Kreutzer S, Wallace E, Asadullah K, et al. Immunopathogenesis of psoriasis. Exp Dermatol. 2007;16:779-98.
11. Lowes MA, Bowcock AM, Krueger JG. Pathogenesis and therapy of psoriasis. Nature. 2007;445:866-73.

12. Menter A, Gottlieb A, Feldman SR, Van Voorhees A, Leonardi CL, Gordon KB, et al. Guidelines of care for the management of psoriasis and psoriatic arthritis: Section 1. Overview of psoriasis and guidelines of care for the treatment of psoriasis with biologics. J Am Acad Dermatol. 2008;58:826-50.

13. Nickoloff BJ, Nestle FO. Recent insights into the immunopathogenesis of psoriasis provide new therapeutic opportunities. J Clin Invest. 2004;113:1664-75.

14. Mackenzie B, Kastelein RA, Cua DJ. Understanding the IL-23 - IL-17 immune pathway. Trends Immunol. 2006;27:17-23.

15. Guttman-Yassky E, Krueger JG. Psoriasis: evolution of pathogenic concepts and new therapies through phases of translational research. Br J Dermatol. 2007;157:1103-15.

16. Whyte HJ, Baughman RD. Acute guttate psoriasis and streptococcal infection. Arch Dermatol. 1964;89:350-6.

17. Gupta MA, Gupta AK, Watteel GN. Early onset (< 40 years age) psoriasis is comorbid with greater psychopathology than late onset psoriasis: a study of 137 patients. Acta Derm Venereol. 1996;76(6):464-6

18. Consenso Brasileiro de Psoríase e guias de tratamento. Rio de Janeiro: Sociedade Brasileira de Dermatologia; 2012.

19. Hurvitz M, Paller AS, Mancini AJ. Papulosquamous and Related Disorders. Hurwitz Clinical Pediatric Dermatology, 4th Edition, Chicago, Editora Elsevier, 2011. p 79-80.

20. Janniger CK, Schwartz RA, Musumeci ML, Tedeschi A, Mirona B, Micali G. Infantile psoriasis. Cutis. 2005;76:173-7.

21. Naldi L, Gambini D. The clinical spectrum of psoriasis. Clin Dermatol. 2007;25:510-18.

22. Morris A, Rogers M, Fischer G, Arch B, Williams K. Childhood psoriasis: review of 1262 cases. Pediatr Dermatol. 2001;18:188-98.

23. Van der Kerkhof P, Schalkwijk J. Psoriasis. In: Bolognia JL, Jorizzo JL, Rapini RP. Dermatology. 2nd ed. New York: Mosby; 2008.

24. Hogan, A. Papulosquamous disease. In: Schachner LA, Hansen RC. Pediatric Dermatology. $3^{\text {rd }}$ ed. Edinburgh: Mosby; 2003. p. 643-6.

25. Beretta-Piccoli BC, Sauvain MJ, Gal I, Schibler A, Saurenmann T, Kressebuch H, et al. Synovitis, acne, pustulosis, hyperostosis, osteitis (SAPHO) syndrome in childhood: a report of ten cases and review of the literature. Eur J Pediatr. 2000;159(8):594-601. 
26. Lewkowicz D, Gottlieb AB. Pediatric psoriasis and psoriatic arthritis. Dermatol Ther. 2004;17:364-75.

27. Lehman JS, Rahil AK. Congenital psoariasis: case report and literature review. Pediatr Dermatol. 2008;25(3):332-338.

28. Gottlieb A, Korman NJ, Gordon KB, Feldman SR, Lebwohl M, Koo JY, et al. Guidelines of care for the management of psoriasis and psoriatic arthritis: Section 2. Psoriatic arthritis: overview and guidelines of care for treatment with an emphasis on the biologics. J Am Acad Dermatol. 2008;58:851-64.

29. Kleinert S, Feuchtenberger M, Kneitz C, Tony HP. Psoriatic arthritis: clinical spectrum and diagnostic procedures. Clin Dermatol. 2007;25:519-23.

30. Mercy K, Kwasny M, Cordoro KM, Menter A, Tom WL, Korman N, et al. Clinical manifestations of pediatric psoriasis: results of a multicenter study in the United States. Pediatr Dermatol. 2013;30(4):424-8.

31. Tollefson MM. Diagnosis and management of psoriasis in children. Pediatr Clin North Am. 2014;61(2):261-77.

32. Romitil R, Maragno L, Arnone M, Takahashi MDF. Psoríase na infância e na adolescência. An Bras Dermatol. 2009;84(1):9-22.

33. Beattie PE, Lewis-Jones MS. A comparative study of impairment of quality of life in children with skin disease and children with other chronic childhood diseases. Br J Dermatol. 2006;155(1)145-51.

34. Ceovic R, Pasic A, Lipozencic J, Murat-Susic S, Skerlev M, Husar K, et al. Treatment of childhood psoriasis. Acta Dermatovenerol Croat. 2006;14:261-4.

35. Darley CR, Cunliffe WJ, Green CM, HutchinsoN PE, Klaber MR, Downes N. Safety and efficacy of calcipotriol ointment (Dovonex) in treating children with psoriasis vulgaris. Br J Dermatol. 1996;135:390-3.

36. Jager ME, de Jong EM, van de Kerkhof PC, Seyger MM. Efficacy and safety of treatments for childhood psoriasis: a systematic literature review. J Am Acad Dermatol. 2010;62(6):1013-30.

37. Cordoro KM. Topical therapy for the management of childhood psoriasis: part I. Skin Therapy Lett. 2008;13:1-3.

38. Pion IA, Koenig KL, Lim HW. Is dermatologic usage of coal tar carcinogenic? A review of the literature. Dermatol Surg. 1995;21:227-31.

39. Hogan A. Papulosquamous disease. In: Schachner LA, Hansen RC. Pediatric Dermatology. 3rd ed. Edinburgh: Mosby; 2003. p. 643-6.

40. Goffe B, Cather JC. Etanercept: an overview. J Am Acad Dermatol. 2003;49(Suppl):S105-11.

41. Paller AS, Siegfried EC, Langley RG, Gottlieb AB, Pariser D, Landells I, et al. Etanercept treatment for children and adolescents with plaque psoriasis. $\mathrm{N}$ Engl J Med. 2008;358:241-51.

42. Northcutt M, Al-Subu A, Bella B, Elitsur Y. Safety of infliximab in children with IBD: the experience of an academic center in WV. W V Med J. 2014;110(3):26-9.

43. Schmeling H, Minden K, Foeldvari I, Ganser G, Hospach T, Horneff G. Efficacy and safety of Adalimumab as first and second used biologic agent in juvenile idiopathic arthritis - the German Biologics JIA Registry (BiKeR). Arthritis Rheumatol. 2014. http://dx.doi.org/10.1002/art.38741.

44. Beattie PE, Lewis-Jones MS. A comparative study of impairment of quality of life in children with skin disease and children with other chronic childhood diseases. Br J Dermatol. 2006;155(1)145-51.

\section{Amanda Hertz}

Setor de Dermatopediatria. Instituto de Dermatologia Professor Rubem David Azulay. Santa Casa da Misericórdia. Rio de Janeiro, RJ, Brasil. 